\title{
Microscale distributions of phytoplankton: initial results from a two-dimensional imaging fluorometer, OSST
}

\author{
Peter J. S. Franks*, Jules S. Jaffe \\ Scripps Institution of Oceanography, University of California San Diego, 9500 Gilman Drive, La Jolla, \\ California 92093-0218, USA
}

\begin{abstract}
A digital camera system was designed to photograph laser-stimulated fluorescence of phytoplankton in situ at $0.67 \times 0.67 \mathrm{~cm}$ resolution over an area of $70 \times 70 \mathrm{~cm}$. In a series of vertical profiles, 1200 images were collected. Criteria were developed to reject images smeared by camera motions (due to ship heave), and images taken when vertical velocities were $>0.2 \mathrm{~m} \mathrm{~s}^{-1}$, leaving 240 good images. Horizontal velocities calculated using ancillary data from the FishTV imaging sonar showed the water to be directed toward the imaging plane during the deployment, supporting the hypothesis that images were uncontaminated by mixing from the instrument package. Images passing all the criteria show strong isotropic spatial variability. The averaged spatial spectrum was flat, suggesting an underlying random distribution of brightly fluorescent particles-probably phytoplankton aggregates. Such observations have important implications for our understanding of the food environment of herbivorous zooplankton, and the mechanisms creating and maintaining patchiness in the ocean.
\end{abstract}

KEY WORDS: Microscale $\cdot$ Patchiness $\cdot$ Fluorescence $\cdot$ Phytoplankton $\cdot$ Fluorometer

\section{INTRODUCTION}

Understanding the horizontal and vertical scales of variability of phytoplankton in the ocean is a necessary step in interpreting the food available to herbivores, and understanding the environment through which they must navigate for food. Numerous studies have concluded that crustaceans and fish larvae were foodlimited at the values of chlorophyll or carbon measured in situ (e.g., review in Owen 1989, Tiselius 1992, Saiz et al. 1993, Cowles \& Fessenden 1995). Many herbivores have been shown to respond to micropatchiness in the laboratory (e.g., Bird \& Kitting 1982, Tiselius 1992, Saiz et al. 1993) and in mesocosms (e.g., Bohrer 1980, Price 1989), and micropatches of food may be necessary to the foraging success of many marine invertebrates and vertebrates.

*E-mail: pfranks@ucsd.edu
The exploration of microscale $(<1 \mathrm{~m})$ structure of plankton in the oceans has been a technology-limited enterprise. Owen (1989) discussed how the advent of the O-ring facilitated the development of in situ profiling fluorometers. The first continuous profiles of phytoplankton fluorescence with microscale resolution (Derenbach et al. 1979) showed strong variability on vertical scales of $10 \mathrm{~s}$ of $\mathrm{cm}$. These thin features were interpreted as layers which might have formed by deceleration of sinking phytoplankton at micropycnoclines. The next major advance came with the development of the laser fluorometer (Cowles et al. 1993, Desiderio et al. 1993), capable of measuring in situ fluorescence emission spectra with centimeter-scale vertical resolution. Again, profiles made with this instrument showed variability at $\sim 10 \mathrm{~cm}$ scales. These thin features were interpreted as being thin layers of phytoplankton, of unknown horizontal extent. A 3-fold increase in fluorescence over 10 to $20 \mathrm{~cm}$ was not unusual in these profiles, confirming similar results ob- 
tained with high-resolution series of bottle samples (Owen 1989, Bjornsen \& Nielsson 1991, Tiselius et al. 1994). Owen (1989), however, interpreted his thin layers as being only about 4 times greater in horizontal extent than vertical, but still not isotropic. Franks (1995) presented a mechanism for the formation of thin layers of phytoplankton through the interaction of existing horizontal patchiness and internal-wavegenerated vertical shear. Based on reasonable shears and the thickness of observed layers, Franks inferred horizontal scales of $<100 \mathrm{~m}$ for $30 \mathrm{~cm}$ thick layers, or $\sim 1 \mathrm{~km}$ horizontal scales for layers $2 \mathrm{~m}$ thick.

Here we present results from the first deployment of a novel in situ fluorescence imaging system, the Optical Serial Section Tomography (OSST) system. This system uses a vertical sheet of laser light to induce fluorescence, which is recorded by a sensitive CCD (charge coupled device) camera. In contrast to other systems, OSST allows the observation of fluorescence emission in a 2-dimensional plane. The system achieved $0.67 \mathrm{~cm}$ resolution over an area of $70 \times 70 \mathrm{~cm}$. Given that this paper describes a new instrument, much of the material in the 'Methods' section is related to the instrument's operation, selection and analysis of the images, and supporting analyses. Readers not interested in the technical aspects of the system are invited to skip to the 'Results and discussion'.

\section{METHODS}

OSST. The OSST uses an argon-ion laser to stimulate fluorescence in a vertical $(x, Z)$ sheet of illumination (Fig. 1). This fluorescence is photographed with a sensitive $1024 \times 1024$ CCD camera (Photometrics, Tuscon, AZ, USA) fixed $1 \mathrm{~m}$ away from the 2 to $3 \mathrm{~mm}$ thick laser sheet. All laser wavelengths below $520 \mathrm{~nm}$ were used to excite fluorescence, and a long-pass (>680 nm) filter was used on the camera. The camera's field of view was $70 \mathrm{~cm} \times 70 \mathrm{~cm}$, and images were binned $10 \times 10$ to improve the signal-to-noise ratio and increase the refresh rate. Each element of the $102 \times 102$ image had a resolution of $0.67 \mathrm{~cm} \times 0.67 \mathrm{~cm}$. The camera's depth of field was made very small $(3 \mathrm{~cm})$ to defocus out-ofplane light at wavelengths $>680 \mathrm{~nm}$ (both fluoresced and scattered). Although this out-of-focus light will contribute to background levels, it will not contribute to observed spatial structures (but see the subsection 'OSST impulse and frequency response', below).

Images were corrected for non-uniform illumination using an internal calibration: the intensity of light at the camera is proportional to the intensity of incident illumination and the concentration of fluorescent material (see Palowitch \& Jaffe 1994, 1995, for a more complete derivation):

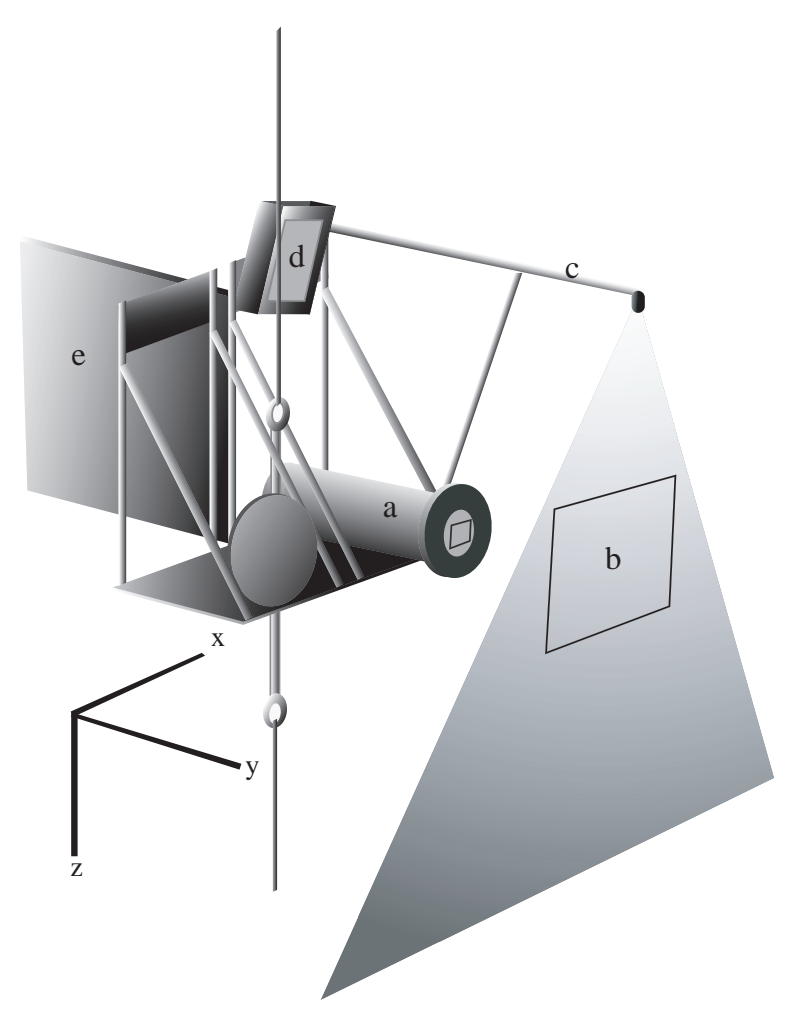

Fig. 1. Schematic of the OSST system showing camera housing (a), imaged area in the laser sheet (b), spar holding the fiber optic lens forming the laser sheet (c), FishTV (d), vane directing the system into the current (e), and coordinate axis. The hydro wire was connected to swivels at the center of the platform

$$
\begin{aligned}
& I_{i}\left(X_{1} Z_{;} r_{2}\right)=\kappa I_{\mathrm{s}}\left(X_{1} Z_{;} r_{2}\right) C\left(X, Z_{i} r_{2}\right) \\
& \exp \left[\int_{r_{1}}-\frac{1}{r_{1}^{2}} C\left(r_{1}, \lambda_{\mathrm{s}}\right) \mathrm{d} r_{1}\right] \exp \left[\int_{r_{2}}-\frac{1}{r_{2}^{2}} C\left(r_{2}, \lambda_{\mathrm{e}}\right) \mathrm{d} r_{2}\right]
\end{aligned}
$$

where $I_{i}\left(x_{1} z_{i} r_{2}\right)$ is the intensity of light in the $(x, z)$ plane recorded by the camera system a distance $r_{1}$ from the source and a distance $r_{2}$ from the camera, $I_{\mathrm{s}}\left(x_{1} Z_{i} r_{2}\right)$ is the intensity of the stimulating wavelength $\lambda_{\mathrm{s}}$ at a point $(x, z)$ in the viewed field, and $\lambda_{\mathrm{e}}$ is the emission wavelength the concentration of the fluorescing compound is $C\left(x, z ; r_{2}\right)$. Fluorescence intensity is reduced by inefficiencies in the fluorescing processes, spherical spreading of light, and camera limitations, all represented by $\kappa$. The first integral term accounts for attenuation of the stimulating beam along its path $r_{1}$ from the source to the point $(x, z)$. The second integral represents absorption of fluoresced light along its path $r_{2}$ from $(x, z)$ to the camera. An additive factor of the camera's dark current, $I_{\mathrm{dc}}$ must also be considered:

$$
I_{o b s}\left(x, Z ; r_{2}\right)=I_{i}\left(x, Z ; r_{2}\right)+I_{\mathrm{dc}}
$$

The raw data must be processed to produce an image in which the intensity of any pixel is proportional to the amount of fluorescing material there (assumed here to be chlorophyll a). We estimated the in-plane concentra- 
tion by first averaging the intensities of all images taken in several vertical profiles (minus the dark current which was collected between each image):

$I_{a v g}\left(X, Z ; r_{2}\right)=\left\langle I_{\mathrm{obs}}\left(X, Z ; r_{2}\right)-I_{\mathrm{dc}}\right\rangle=\left\langle\kappa I_{\mathrm{s}}\left(X, Z ; r_{2}\right) C\left(X, Z ; r_{2}\right)\right.$

$\exp \left[\int_{r_{1}}-\frac{1}{r_{1}^{2}} C\left(r_{1}, \lambda_{\mathrm{s}}\right) \mathrm{d} r_{1}\right] \exp \left[\int_{r_{2}}-\frac{1}{r_{2}^{2}} C\left(r_{2}, \lambda_{e}\right) \mathrm{d} r_{2}\right]>$

This gives the average attenuation of stimulating and fluoresced irradiance along the 2 paths. Dividing (1) by (3) yields

$$
I_{\text {comp }}=I_{i}\left(X, Z ; r_{2}\right) \propto C\left(X, Z ; r_{2}\right)
$$

This procedure assumes that the relative attenuation of incident light across the stimulated plane can be accurately approximated by a mean value which is a function of 2 dimensions: the $(x, z)$ illumination plane. This appears reasonable because the stimulating light is blue-green, and both absorption and scattering are small over the $1 \mathrm{~m}$ vertical path the light must travel to stimulate fluorescence at the bottom of the image (the laser sheet shone downward). It was also assumed that variation of attenuation of emitted light could be modelled by its mean value over the field of view. Unfocused sources of light (such as scattered light) can be regarded as virtual sources; their images will be spread over the entire field of view, producing only a mean value for the entire image. On the other hand, differential absorption (in the red) of emitted light by compounds between the camera and the illuminated sheet could lead to local changes in recorded intensity. To estimate these error sources, we assume a chlorophyll (chl) concentration between the camera and

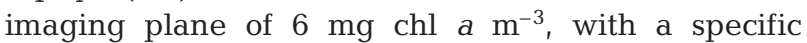
absorption coefficient of $0.02 \mathrm{~m}^{2} \mathrm{mg}^{-1}$ (e.g., Morel 1988 and references therein). This yields an attenuation coefficient of $0.12 \mathrm{~m}^{-1}$. Over the $1 \mathrm{~m}$ path length between the camera and imaging plane, this would give an attenuation of about $12 \%$. Doubling this attenuation coefficient still only gives an attenuation of $21 \%$. This change is much smaller than the factors of 2 to 10 variability we measured over small regions of any image. Thus we are confident that the relative local variations of fluorescence we measured are real.

Deployment. The camera was housed in a pressure case $35 \mathrm{~cm}$ in diameter and $70 \mathrm{~cm}$ long. The camera housing was fixed to a rectangular scaffolding $1.5 \mathrm{~m}$ tall and $1 \mathrm{~m}$ wide, which also held the vane to keep the instrument pointed into the current, and swivels to attach to the hydrowire at the top, and a wire with the weight (train wheels) at the bottom. The weight hung approximately $80 \mathrm{~m}$ below the camera package. The laser light sheet was $1 \mathrm{~m}$ upstream of the package; the rod holding the fiber optic light source was the only flow-deflecting structure upstream of the camera. Calculations pre- sented below confirm that the instrument package was oriented into the flow. Flow-tank tests of a scale model of a similar instrument platform confirmed that the system did not disturb the water in the imaging plane.

In addition to the OSST system, the instrument package held a pressure sensor and a 3-dimensional imaging sonar system called FishTV (Jaffe et al. 1995, McGeehee \& Jaffe 1996, Jaffe 1999). The FishTV used 2 arrays of eight $445 \mathrm{kHz}$ transducers to divide the imaging volume (approximately $6 \mathrm{~m}^{3}$ ) into $8 \times 8$ horizontal and vertical bins, with the range away from the instrument forming the third dimension. The OSST imaging volume was included in the FishTV imaging volume (Jaffe et al. 1998). The FishTV can detect and track acoustic targets $>1 \mathrm{~cm}$ in length, such as euphausiids and fishes. Here we use the FishTV data to quantify the horizontal velocities to or from the OSST.

To calculate the volume of water imaged by the OSST, we use the width of the laser sheet (2 to $3 \mathrm{~mm}$ ), the exposure time (30 ms, as given by the camera manufacturer) and the speed of water past the instrument ( $20 \mathrm{~cm} \mathrm{~s}^{-1}$, estimated from the FishTV acoustics, see subsection 'Selection of images: velocity' below). This gives a thickness of the image of $\sim 1 \mathrm{~cm}$, and a total volume imaged of $70 \times 70 \times 1 \mathrm{~cm}$ or $4900 \mathrm{~cm}^{3}$. The sampling rate of the OSST was $0.9 \mathrm{~Hz}$.

Observations were made from the RV 'Sproul', moored in $300 \mathrm{~m}$ of water 30 nautical miles west of the Scripps Institution of Oceanography (San Diego, California) on July 25 to 28, 1995. The instrument package, consisting of the FishTV, OSST, depth and temperature sensors was used to profile between 20 and $80 \mathrm{~m}$. Casts were made with a Sea Bird SBE-19 CTD integrated with a Wet Labs Wet Star fluorometer and a SeaTech $25 \mathrm{~cm}$ path length transmissometer approximately $1 \mathrm{~h}$ prior to and after the OSST deployment. The OSST data discussed below were obtained from a series of 4 vertical profiles (down-up-down-up) taken at night between 20:40 and 21:40 h local time, July 27.

Selection of images: smearing. The instrument package was profiled vertically between 20 and $80 \mathrm{~m}$, with a nominal speed of $0.5 \mathrm{~m} \mathrm{~s}^{-1}$. The profiling speed was not constant, however, due to the heave of the ship with a period of 4 to $5 \mathrm{~s}$. This variable speed led to obviously smeared images as the package moved suddenly between the crest and trough of a wave. As will be shown below, the package was practically stationary at the crests and troughs of the waves.

To diagnose and remove smeared images, an estimate of the 2D image spectrum was calculated using Welch's (1967) method. A center block of $96 \times 96$ pixels was divided into a set of $5 \times 5$ overlapping images of $32 \times 32$ pixels which were each multiplied by a Hanning window. The amplitudes of the set of 25 Fourier transform coefficients were then averaged (Fig. 2). 

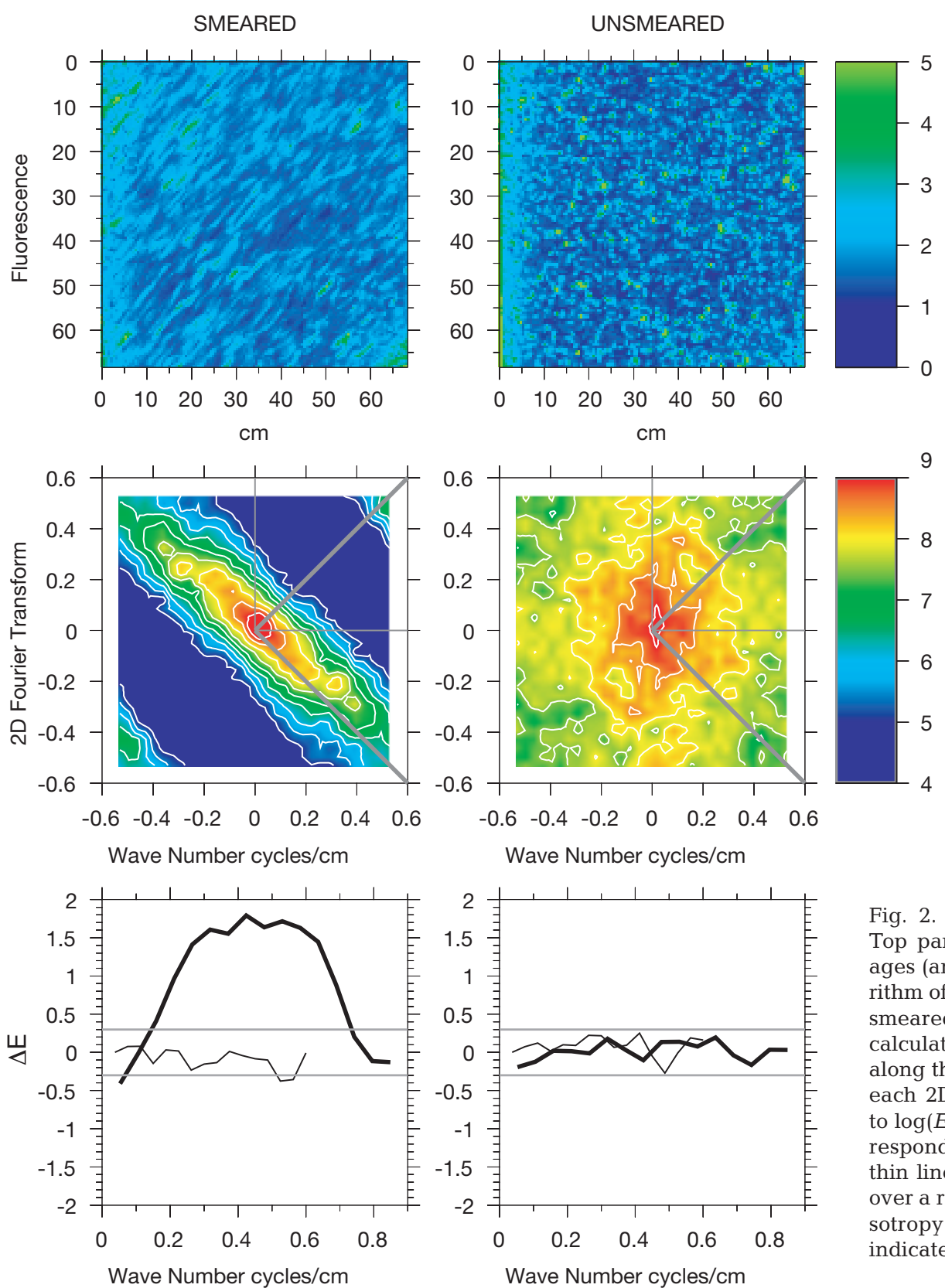

Fig. 2. Identification of smeared images. Top panels: smeared and unsmeared images (arbitrary units). Middle panels: logarithm of the 2D spectra of smeared and unsmeared images. Bottom panels: $\Delta E$ (Eq. 5) calculated from the ratios of $1 \mathrm{D}$ spectra along the 2 thick lines or the 2 thin lines in each 2D spectrum; thick lines correspond to $\log \left(E_{1} / E_{2}\right)$ of the 2 thick lines in the corresponding $2 \mathrm{D}$ spectrum; similar for the thin lines (see Eq. 5); large or small ratios over a range of wavenumbers indicate anisotropy (smearing) of images; gray lines indicate $\Delta E=\log \left(E_{1} / E_{2}\right)= \pm 0.3$, or $E_{1} / E_{2}=$ $0.5,2$ (see Eq. 5)

In an isotropic image, the 2D spectrum should be radially symmetric, while in an anisotropic image, the 2D spectra form elongate ellipses oriented with the major axis perpendicular to the direction of smearing (Fig. 2). Smeared images had low variance at high wavenumbers along the axis of the smearing compared to lines perpendicular to the axis of the smearing. To objectively diagnose smearing, 2 perpendicular pairs of 1D spectra were chosen from the 2D transform. The first pair were vertical and horizontal, the second pair were diagonals perpendicular to each other. The ratio of the variance at a given wavenumber was cal- culated for the 2 members of a pair. If an image were perfectly isotropic, the ratio of the 2 perpendicular spectra would be about 1 (with some variation due to inherent variability in the images). In an anisotropic image, the ratio would be larger or smaller than 1.

The criteria used to diagnose smearing were:

$$
\frac{1}{k_{\max }} \sum_{n=1}^{k_{\max }} \frac{E_{1}\left(k_{n}\right)}{E_{2}\left(k_{n}\right)}<0.5 \text { or } \frac{1}{k_{\max }} \sum_{n=1}^{k_{\max }} \frac{E_{1}\left(k_{n}\right)}{E_{2}\left(k_{n}\right)}>2
$$

where $E_{1}$ is the variance at wave number $k_{n}$ of the first member of the pair of 1D spectra, $E_{2}$ the variance of the second member of the same pair, and $k_{\max }$ the total 
number of wavenumbers. This value was calculated for both pairs of 1D spectra. If one pair failed the test (i.e., the average of the ratios was $<0.5$ or $>2$ ), the image was rejected and not used in further analyses. (Note that if the smearing is oriented in the direction of one of the 1D spectra, the second pair will not show any effect. It would be unlikely, however, for both pairs to miss the smearing.) The average over all wavenumbers was taken to account for incoherent anisotropies in images: long patches might occasionally lead to large differences at one wavenumber, but not to consistent differences over several wavenumbers. These criteria ensure that the ratio of the major to minor axes of the 2D transform ellipses was less than a factor of 2 .

Images with low variance $(<0.5)$ were also rejected, since the signal-to-noise ratio in these images was low. Such images appeared above and below the chlorophyll maximum layers $(<30 \mathrm{~m},>68 \mathrm{~m})$. After applying the smearing and low-variance criteria, 412 good images were left from the original 1200.

Selection of images: velocity. The camera shutter speed was given by the manufacturer as $30 \mathrm{~ms}$. With a pixel size of $0.67 \mathrm{~cm}$, a motion of the camera $>20 \mathrm{~cm} \mathrm{~s}^{-1}$ will lead to smearing over more than 1 pixel distance. As a third criterion for selecting good images, the vertical velocities of the instrument package were calculated and all images with speeds $>20 \mathrm{~cm} \mathrm{~s}^{-1}$ were rejected.

The vertical velocities $w$ were calculated from the depth sensor on the instrument package:

$$
w(i)=0.5\left(\frac{z(i-1)-z(i)}{t(i-1)-t(i)}+\frac{z(i)-z(i+1)}{t(i)-t(i+1)}\right)
$$

where $z(i)$ is the depth of the image being considered, $z(i-1)$ is the depth of the previous image, and $z(i+1)$ is the depth of the next image (similarly for time, $t$ ). This criterion was quite stringent (Fig. 3), and led to the rejection of a further 172 images, leaving a total of 240 good images. The retained images were almost exclusively at the peak or trough of the wave cycle on both the upcasts and downcasts.

Horizontal velocities. Since the focus of this study was small-scale $(1 \mathrm{~cm}$ to $1 \mathrm{~m})$ structures of fluorescence, it is necessary to ensure that the observed structures did not derive from mixing generated by the instrument package itself. The package was fitted with a vane to keep it oriented into the ambient flow. If the water velocities were constantly pointed into the package (and thus the laser sheet), there should be relatively little disturbance of the imaged water by the instrument package, as confirmed by our flow-tank tests. While there were no current meters on the system, the acoustic images gathered by the FishTV can be used to calculate velocities directed toward the transducers.

The FishTV was used to calculate the horizontal displacement of particles between successive acoustic images gathered at $2 \mathrm{~Hz}$. A similar technique was used by Sutton \& Jaffe (1992) to estimate bedload velocity. FishTV produces an $8 \times 8 \times 512$ matrix of acoustic backscatter for each sonar frame. Defining a coordinate system where $r$ is range from the sonar and $x$ and 
$z$ are the lateral distances (similar to Fig. 1), the complex data were first converted to a real array by computing the magnitude of each array element and then integrating in the $x$ and $z$ directions to yield a 1-dimensional vector aligned along the $r$ axis (away from the FishTV). A section of this array was extracted, extending between 2.3 and $3.3 \mathrm{~m}$ away from the sonar (128 values, $\sim 0.8 \mathrm{~cm}$ resolution in range), using only the inner $2 \times 2$ beams. This short distance minimizes the effects of beam spreading and attenuation. Next, successive waveforms were cross-correlated and the maximum value of the cross-correlation was used to estimate the optimal value of the inter-waveform shift (the distance moved by the particles). This estimate of particle motions was not reliable when large translations of the instrument package changed the field of view completely between successive frames. In order to address this problem an estimate of difference was computed between the 2 waveforms that were used to compute each of the data shifts by computing an inner product between the $1 \mathrm{D}$ vectors. Then, a threshold was used as a criterion for data acceptance. This value was adjusted empirically until unreasonable values of the estimated current were eliminated. A minimum value of 0.9925 for the inner product (coherence between waveforms) was found to yield profiles which were smooth and devoid of physically unrealistic values. Throughout the procedure, the ratio of positive to negative values of the acceptable mean-square errors was nearly constant, indicating that it was unlikely that there was any bias introduced by our ad hoc measure for characterizing acceptable data.

The FishTV transducers were mounted on the instrument package pointing downward at $13^{\circ}$ from the horizontal. On the upcast, the horizontal component of the velocity toward the FishTV is underestimated because the vertical velocity gives a component of the velocity away from the acoustic system. The amount by which the actual horizontal velocity was underestimated is proportional to $1 / \cos \left[\tan ^{-1}(w / V)+13^{\circ} \pi / 180^{\circ}\right]$, where $w$ is the vertical velocity and $v$ the actual horizontal velocity. If the vertical and horizontal velocities were equal, we would underestimate the horizontal velocity by about $25 \%$. To be conservative, all horizontal velocities from the upcasts were multiplied by 1.15 . On the downcasts the horizontal velocities were overestimated by an amount proportional to $1 / \cos \left[\tan ^{-1}(w / V)-\right.$ $13^{\circ} \pi / 180^{\circ}$ ]. For $w=V$, this is about $20 \%$. Therefore the downcast data were multiplied by 0.8 .

A plot of horizontal velocities versus depth (Fig. 4) confirms that water was flowing toward the camera plane, with speeds of 5 to $30 \mathrm{~cm} \mathrm{~s}^{-1}$. The higher speeds were obtained deeper in the water column (>55 m). A total of 11 records out of 243 good velocity records indicated velocities directed away from the camera

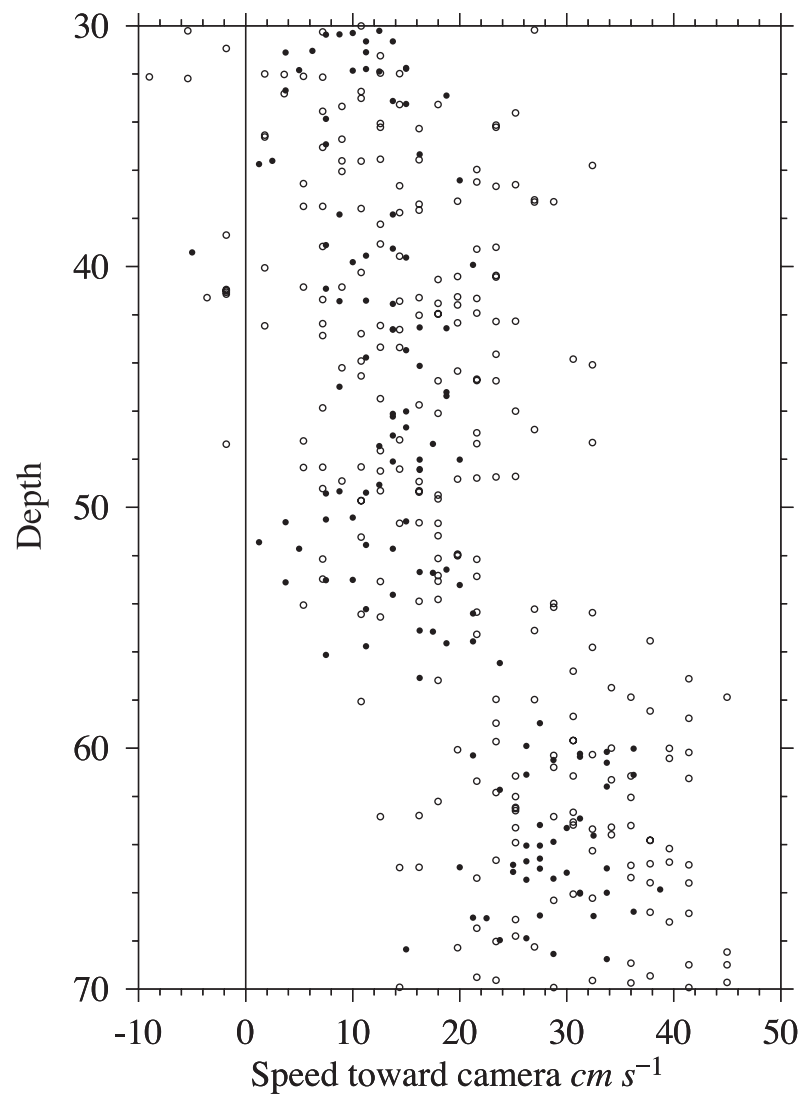

Fig. 4. Horizontal water speeds toward the camera calculated from the FishTV acoustics. Negative values are away from the camera (indicating possible contamination of an image by the wake of the instrument package). ( $($ ) Datapoints obtained on the upcast; $(\bullet)$ datapoints from the downcast. Each value represents $0.5 \mathrm{~s}$ (the time between acoustic images). No negative values were obtained below $50 \mathrm{~m}$

system, all at speeds $<8 \mathrm{~cm} \mathrm{~s}^{-1}$, and never for more than $0.5 \mathrm{~s}$. Most of these were obtained on the upcasts (9 of 11 occurrences), and all were at depths $<50 \mathrm{~m}$. The horizontal speeds were slightly higher on the downcasts than the upcasts, confirming our underestimate of the horizontal velocities on the upcasts.

Since the water was flowing constantly toward the laser sheet at a fairly high speed, it was assumed that the images were uncontaminated by mixing induced by the instrument package. The high acoustic waveform correlations are additional evidence that the platform was not turbulently mixing the water at these ranges, as the correlations would be quite low if the water was being mixed. Note also that other platform motions such as rotation about the axis of the cable and pitching are orthogonal to the current direction computed here, and thus do not bias the data. Motions of the sonar along the $y$ axis were unlikely, so that the data represent actual values of the horizontal current at the study site. 
OSST impulse and frequency response. To interpret the spatial variability of fluorescence in the images, it is necessary to calculate the system's impulse response and frequency response-the filter characteristics of the instrument. If the instrument were working perfectly, each pixel would be completely independent of its neighbors. However, the system is never perfect, and the information at one pixel is weighted with information from adjacent pixels. The degree of weighting is known as the impulse response, while the Fourier transform of the impulse response is the frequency response. The frequency response gives the relative attenuation of variance at each wavenumber, i.e., the filter characteristics of the system (e.g., high-pass, low-pass, etc.). To calculate the impulse response, we searched through the good images for isolated bright pixels, assuming that these were fluorescent particles smaller than the resolution of the camera (delta functions in terms of the camera); 25 such pixels were found. Their peaks were aligned, and an average radial fluorescence profile was calculated along the 2 adjacent horizontal and vertical pixels (Fig. 5a). This 1-dimensional average peak was used as a convolution kernel to determine the system's frequency response. Note that the frequency response is reasonably sensitive to the exact choice of kernel-slight changes in the values could lead to relatively large changes in the frequency response. A Montecarlo simulation was performed, convolving the impulse response with random time series, and calculating their spectra (Fig. 5b). The frequency response showed the system to behave as a weak low-pass filter: variance decreased at high wavenumbers (small spatial scales). The roll-off began at 5 to $10 \mathrm{~cm}$ scales, with $\sim 60 \%$ reduction in variance at $1 \mathrm{~cm}$ scales. With this frequency response, an underlying white spectrum would be distorted by our camera system to resemble the spectrum of Fig. 5b-decreasing variance with increasing wavenumber. The standard deviations shown in Fig. $5 \mathrm{~b}$ are conservative, given that the variance in the impulse response kernel was not included in these estimates.

\section{RESULTS AND DISCUSSION}

The CTD/fluorometer profiles taken $\sim 1 \mathrm{~h}$ prior to and $\sim 1 \mathrm{~h}$ after the OSST profiles showed a relatively linear increase of density with depth (Fig. 6). The steppy structure was driven by temperature; within the resolution of our CTD, no evidence was found for extensive overturns or mixing. The facts that the density profile is quite linear, and the images were acquired well below the seasonal pycnocline at $15 \mathrm{~m}$, suggest that the water column was not particularly turbulent.
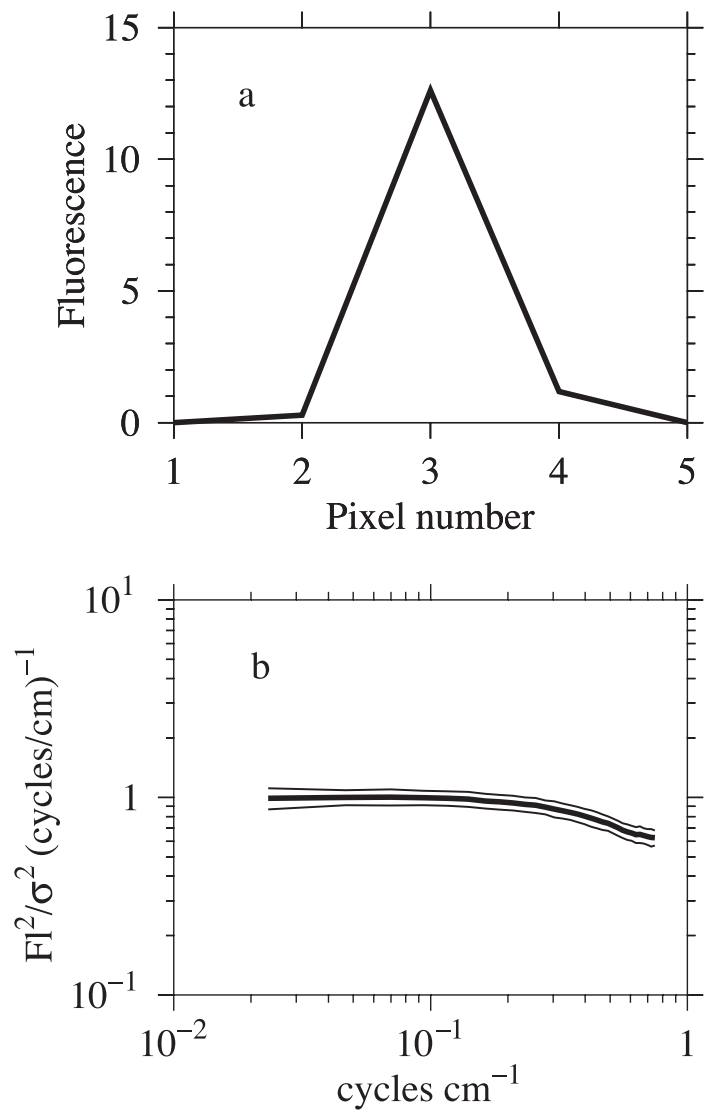

Fig. 5. System (a) impulse and (b) frequency response. If the system were perfect, there would be no fluorescence $\left(\mathrm{Fl}_{i}\right.$ arbitratry units) at Pixels 1, 2, 4 and 5, only a spike at Pixel 3. The frequency response shows the system acts as a weak low-pass filter, reducing the high-wavenumber (short wavelength) variance by as much as $40 \%$. The standard deviations plotted in (b, thin lines) are asymmetric about the mean, although they may appear otherwise (see also Fig. 11)

The fluorescence showed 2 peaks: a broad primary maximum between 45 and $60 \mathrm{~m}$, and a thinner, intense secondary maximum between 60 and $68 \mathrm{~m}$. This secondary maximum appeared and disappeared from our study site over about $10 \mathrm{~h}$. Analyses of rates of increase of chlorophyll suggest that this feature was probably a patch that advected past our anchor station. The plots of fluorescence versus $\sigma_{\mathrm{T}}$ (Fig. 6) show that the fluorescence peaks remained on their isopycnals (to within the resolution of our CTD) throughout the deployment, even though they moved up and down in space. The 4 vertical profiles with the OSST were taken over about $1 \mathrm{~h}$, and varied little except for offsets due to internal waves.

A total of 240 images passed the selection criteria, and will be referred to as 'good' images. A histogram of the frequency of occurrence of good images versus depth (Fig. 7) shows the images to be relatively evenly spread between 30 and $68 \mathrm{~m}$ depth. Between 5 and 20 

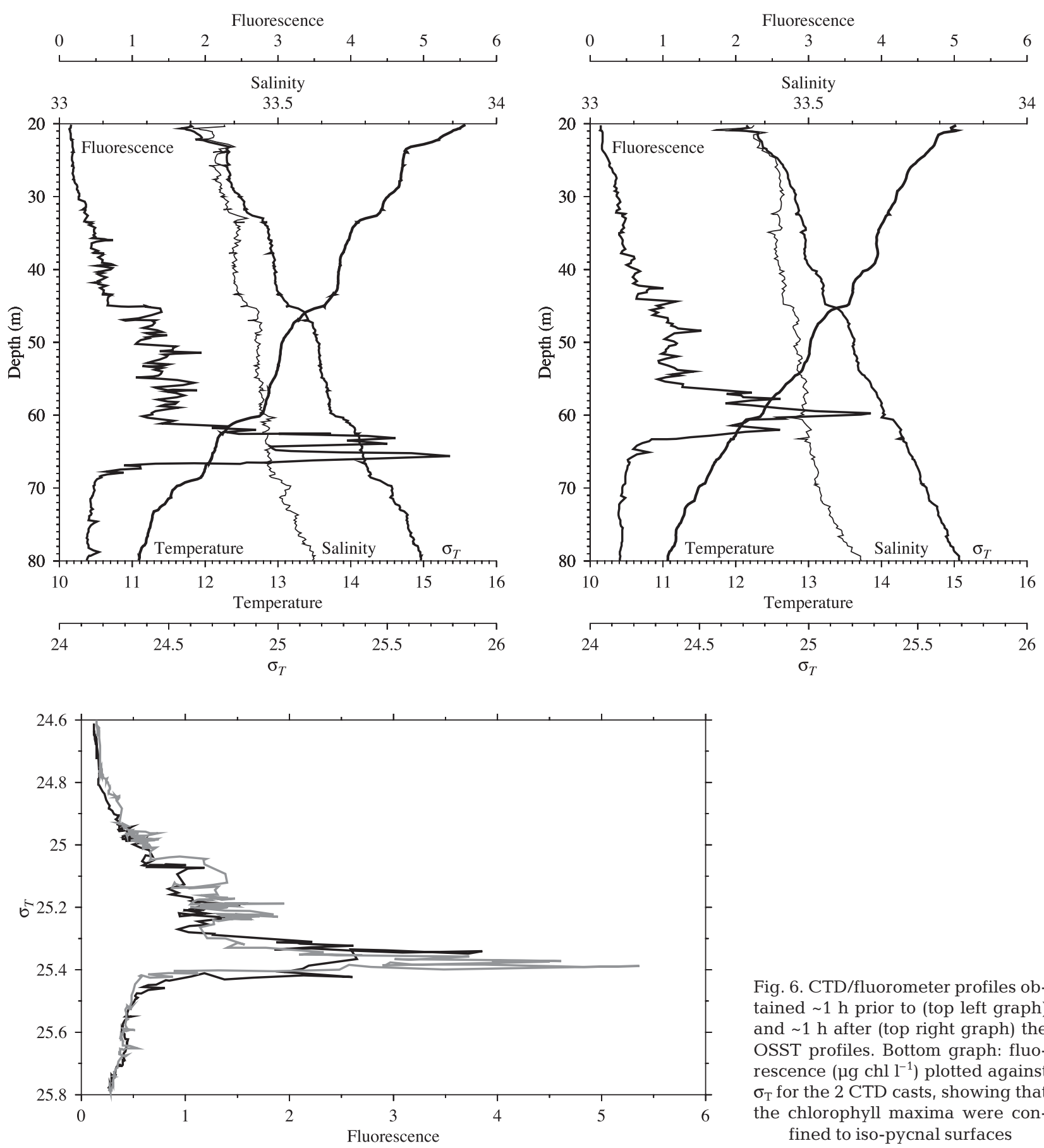

Fig. 6. CTD/fluorometer profiles obtained $\sim 1 \mathrm{~h}$ prior to (top left graph) and $\sim 1 \mathrm{~h}$ after (top right graph) the OSST profiles. Bottom graph: fluorescence $\left(\mu \mathrm{g} \mathrm{chl} \mathrm{l}^{-1}\right)$ plotted against $\sigma_{\mathrm{T}}$ for the 2 CTD casts, showing that the chlorophyll maxima were confined to iso-pycnal surfaces

good images were found in each $2 \mathrm{~m}$ bin. The lowvariance criterion rejected most of the images $<30$ and $>68 \mathrm{~m}$ where the fluorescence was low.

To calculate an approximate calibration curve for the OSST, fluorescence values from the WetStar fluorometer were compared to the OSST values (Fig. 8). Two WetStar profiles were used, the first obtained about $1 \mathrm{~h}$ prior to the OSST profiles, the second about $1 \mathrm{~h}$ after. Fluorescence values were averaged into $2 \mathrm{~m}$ bins. The average fluorescence value for an OSST image was used in the comparison. As noted in 'Methods', this average covers approximately 4.91 of water. Both CTD casts give statistically identical calibration curves, although more variance is explained by the first cast $\left(\mathrm{r}^{2}=\right.$ 0.56 for the combined casts). Sharp gradients in properties, combined with smearing in the tubing leading to the WetStar and depth offsets due to internal waves, lead to imperfect alignment of the various profiles and 


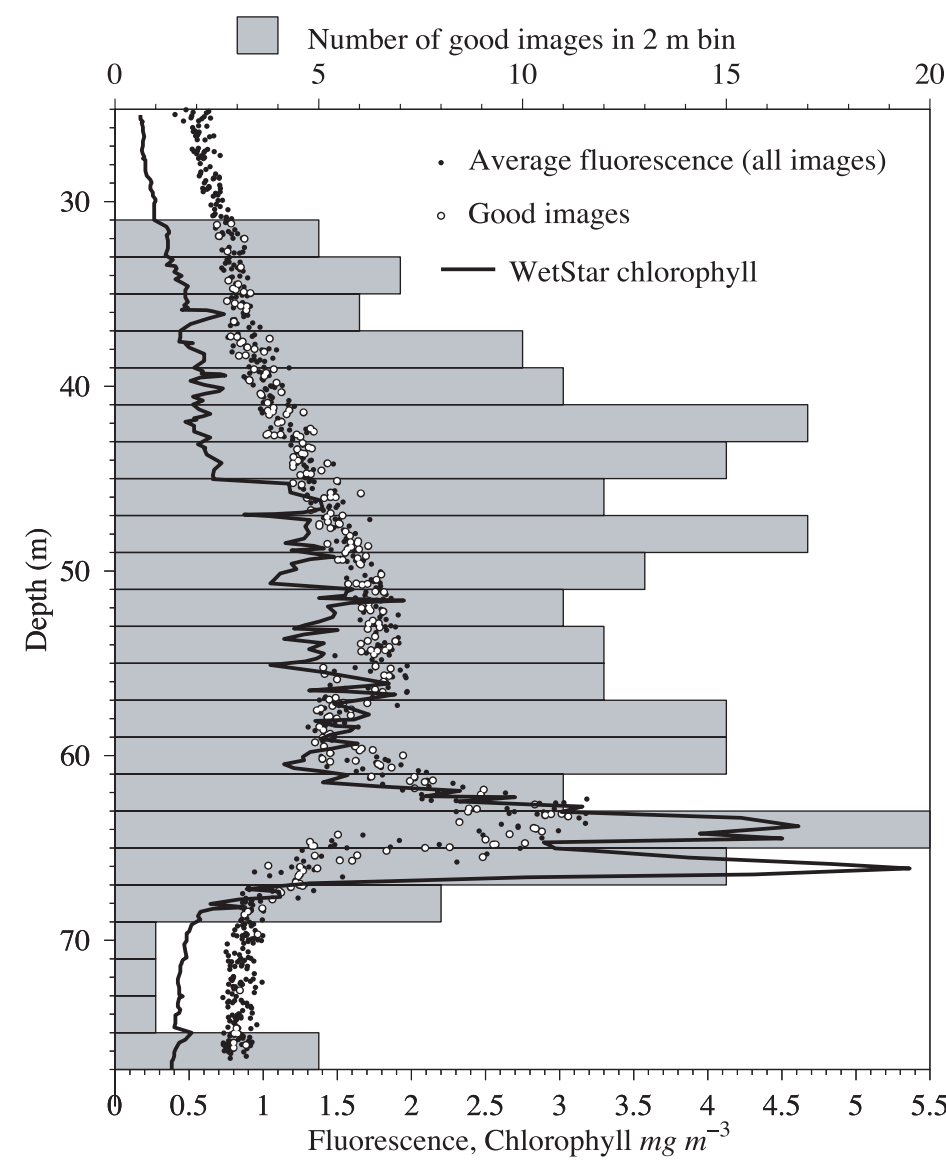

Fig. 7. Histogram, top axis: number of good images in each $2 \mathrm{~m}$ bin between 31 and $77 \mathrm{~m}$; continuous line, bottom axis: fluorescence $(\mathrm{mg} \mathrm{chl}$ $\mathrm{l}^{-1}$ ) from a vertical profile of a WetStar fluorometer $\sim 1 \mathrm{~h}$ prior to the OSST deployment; $(\bullet)$ average fluorescence (arbitrary units) from all 1200 OSST images (note that the average is not affected by smearing),

(O) average fluorescence from 240 good OSST images

contributes to variability in the calibration curve. The calibration fits of Fig. 8 suggest that the OSST was imaging average chlorophyll concentrations down to $0.3 \mathrm{mg} \mathrm{m}^{-3}$, and up to $5 \mathrm{mg} \mathrm{m}^{-3}$.

We are reasonably certain that the fields imaged by the OSST were uncontaminated by the instrument itself. This assertion is based on several lines of evidence: (1) The imaging plane (laser sheet) was $1 \mathrm{~m}$ upstream of the camera package and frame. (2) The FishTV showed the horizontal velocities to be directed toward the camera package. The horizontal velocities were typically $>10 \mathrm{~cm} \mathrm{~s}^{-1}$ toward the package. Thus mixing of water in the imaging plane would have to have occurred $>1 \mathrm{~m}$ away from the package. The high waveform correlations of the FishTV data show that the water was not mixing upstream of the package. (3) Only a wave could propagate against the current to mix water at the imaging plane. The instrument package oscillated with a frequency $\gg \mathrm{N}$, the buoyancy fre- quency. The package would thus generate turbulence, not internal waves. The mixed water would advect away from the imaging plane in the direction of the ambient flow. (4) Flow-tank tests with a scale model showed that the package did not disturb the flow except within millimeters of the frame, even when the frame was oscillating. We are thus confident that the images passing the selection criteria show the spatial patterns of fluorescence as they existed in situ.

One of our main motivations in designing OSST was the study of the microscale spatial variability of fluorescence. Fig. 9 shows 4 good images, representative of 4 regions of the water column: above the chlorophyll maxima (31.86 m), in the shallow chlorophyll maximum $(53.13 \mathrm{~m})$, in the deeper maximum $(64.72 \mathrm{~m})$, and below the maxima $(68.43 \mathrm{~m})$. Visual inspection of these images reveals a high degree of patchiness of fluorescence. A section through the image from $64.72 \mathrm{~m}$ (Fig. 10) shows changes in fluorescence of almost an order of magnitude over a few pixels distance. These changes are far greater than could be accounted for by patchiness of material between the imaging plane and the camera (as discussed above), and we interpret them to be real variability in fluorescence. The images present a 'starry' view of fluorescence patchinessimages appear to show many small, isolated regions of relatively intense fluorescence with little structure between these regions. The number and intensity of these regions increases in the fluorescence maxima. The left-hand edge and

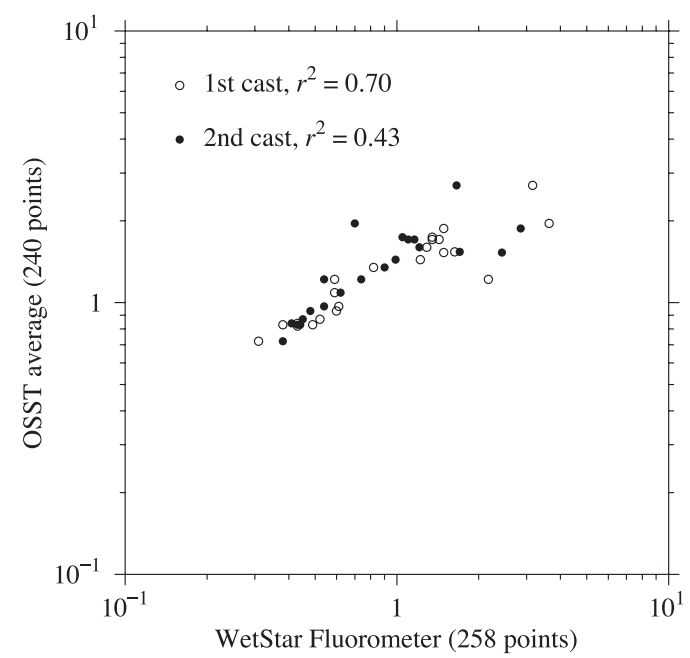

Fig. 8. Relationship of OSST fluorescence (arbitrary units) and chlorophyll $\left(\mathrm{mg} \mathrm{m}^{-3}\right)$ estimated from the WetStar fluorometer. Data points are averages of $2 \mathrm{~m}$ bins. Two WetStar casts are shown, $\sim 1 \mathrm{~h}$ prior to, and $\sim 1 \mathrm{~h}$ after the OSST deployment (see Fig. 6) 
$31.86 \mathrm{~m}$
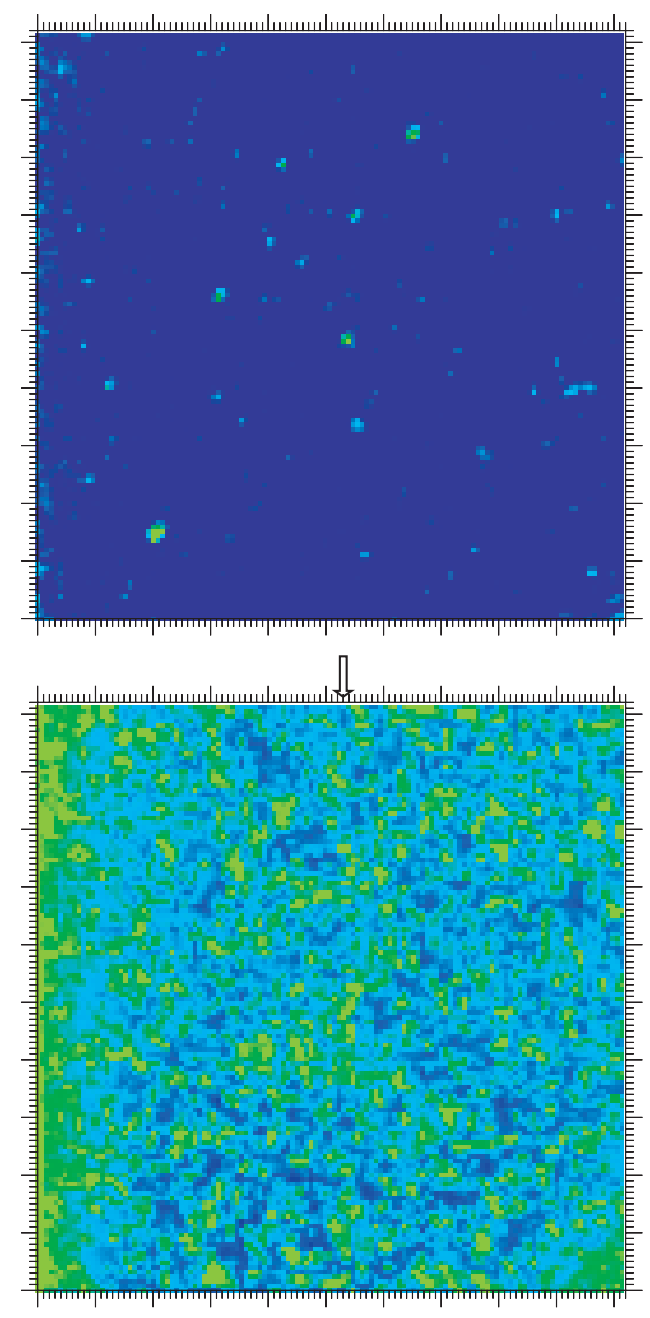

$64.72 \mathrm{~m}$
$53.13 \mathrm{~m}$
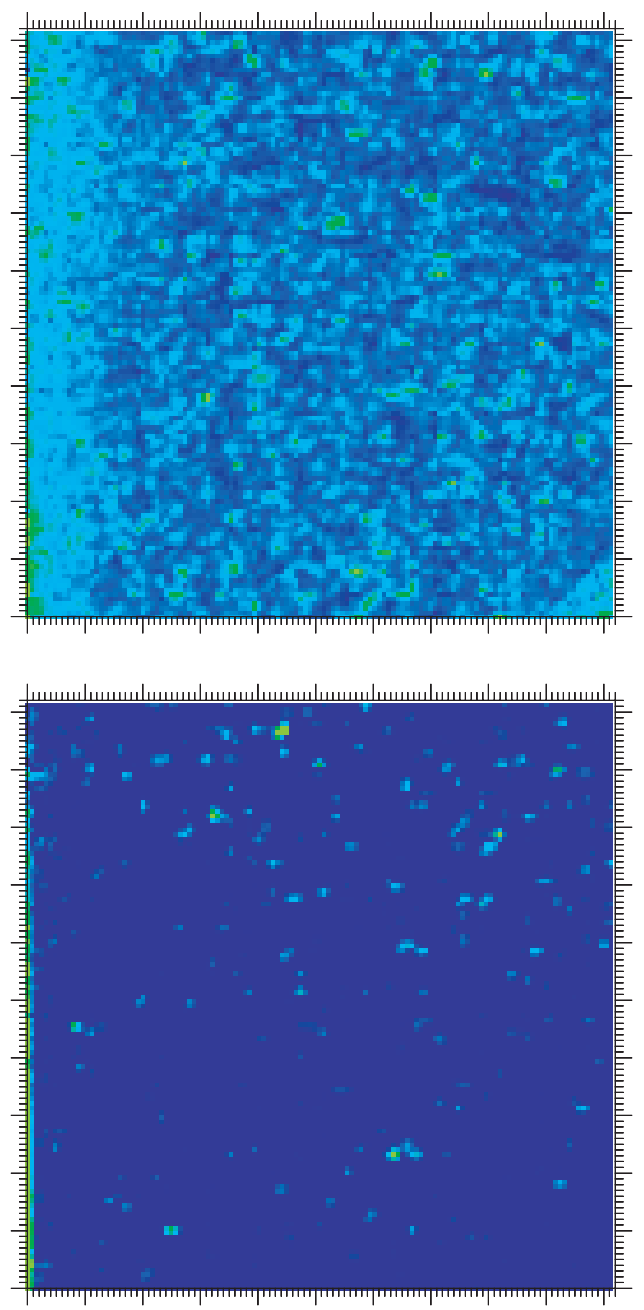

$68.43 \mathrm{~m}$

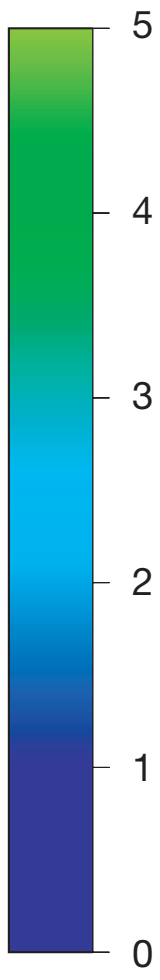

Fig. 9. Four good images of laser-induced fluorescence (arbitrary units) from representative regions of the water column (see Fig. 6 for CTD/fluorometer profiles). Arrow above $64.72 \mathrm{~m}$ image indicates the slice in Fig. 10. Color bar in OSST units (see Fig. 8) lower-right corner of the images show the edge of the laser sheet; these regions were not used in any of the analyses. By rejecting smeared images (criterion given in 'Methods'), images with naturally-formed layers in them might also be rejected. After checking every rejected image, it was apparent that no obviously layered images were rejected by this criterion, although some images had obvious gradients in the number of bright pixels from the top to the bottom of the image, particularly images captured at the upper and lower edges of the secondary maximum (e.g., Fig. 9: 68.43 m).

Knowing the system impulse response, it is possible to interpret spatial spectra calculated from the images. Such spectra should contain important information on the spatial variability of fluorescence, and potential clues concerning the dynamics underlying its formation. To address the relationship of fluorescence variability with spatial scale, 64 vertical and 64 horizontal
1D spatial spectra were calculated from a $64 \times 64$ pixel subimage in the center of each good image. No statistical differences were found between the average vertical and horizontal spectra (i.e., the variability was isotropic). The vertical and horizontal spectra were therefore averaged to obtain a grand average for each image. This average 1D spectrum was divided by the total variance of the subimage. If the different images have the same spatial structures but different fluorescence intensities, this scaling should allow the spectra to lie on top of each other. This was the case (Fig. 11), as the mean \pm standard deviation of the 240 average spectra form a tight grouping.

The similarity between the averaged spatial spectrum calculated from the data (Fig. 11) and the system frequency response (Fig. 5b) is striking. Statistically, the 2 spectra are basically indistinguishable, with broad overlap of the standard deviations at each wave- 


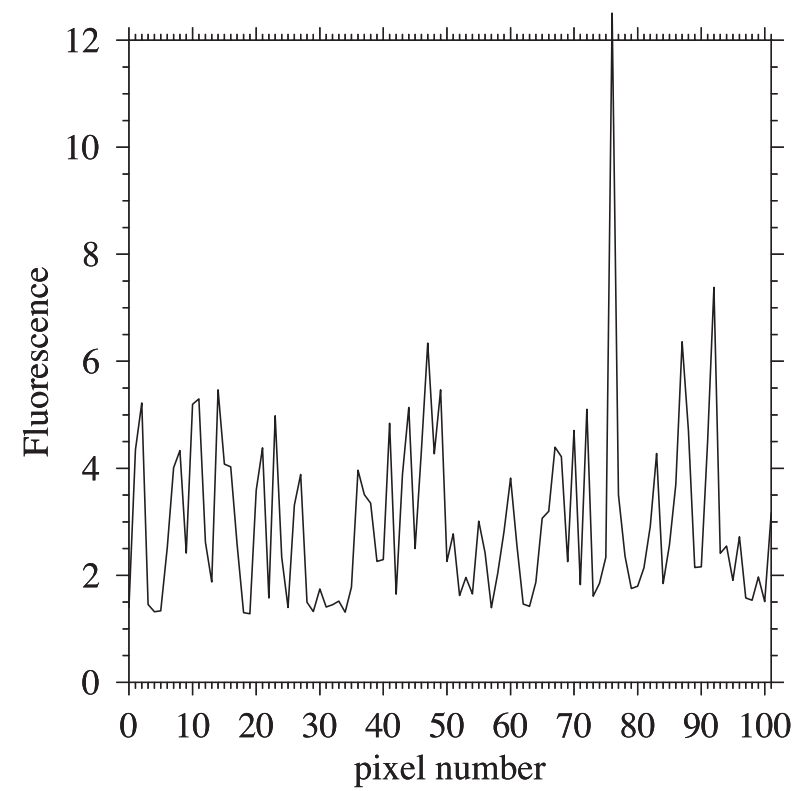

Fig. 10. A slice through a good image (arrowed in Fig. 9). Variability of an order of magnitude over 1 or 2 pixels was not uncommon (arbitrary units)

number. Since the system's frequency response (Fig. 5b) shows how the system would alter a white spectrum, and since the observed average spectrum (Fig. 11) is statistically no different than the system frequency response, it is fair to conclude that the true

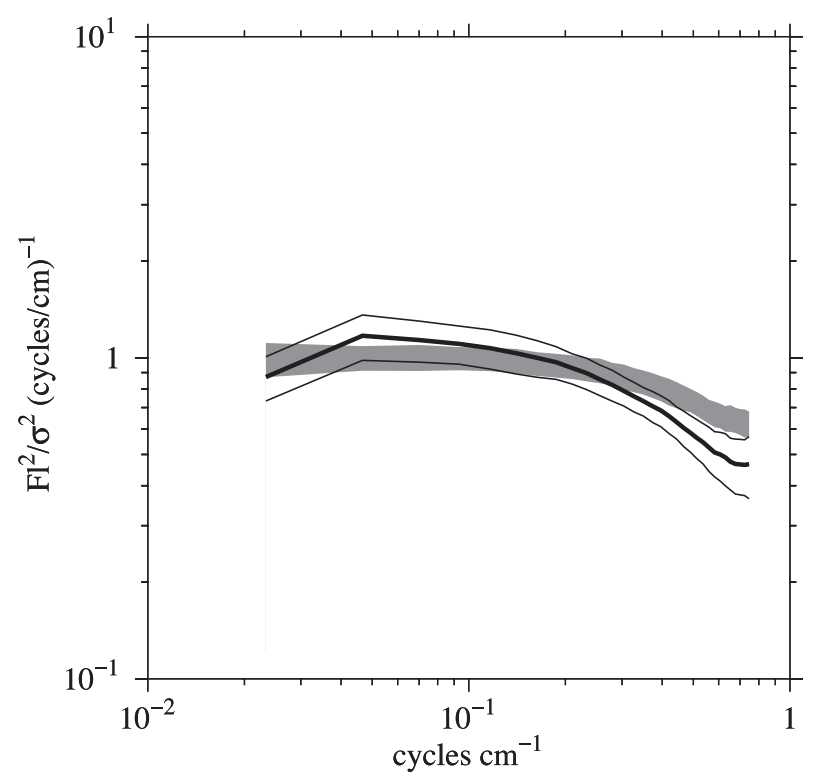

Fig. 11. Mean \pm standard deviation of spatial spectra from the 240 good images; 68 horizontal and 68 vertical spectra were calculated from a $68 \times 68$ subimage of each image, and averaged. Spectra were normalized by the variance of each subimage. The thick gray line is the area bounded by $\pm 1 \mathrm{SD}$ of the system frequency response (Fig. 5b) spatial spectrum of fluorescence variance in our images is white-equal variance at all scales $<70 \mathrm{~cm}$.

A white spectrum of fluorescence variance implies that there is no spatial autocorrelation between pixels in an image. One physically realistic way of generating a white spectrum that appears consistent with our data is an underlying Poisson spatial distribution of isolated bright fluorescent points, each smaller than the resolution of the camera $(0.67 \times 0.67 \mathrm{~cm})$. The number and intensity of the points would affect the power (vertical offset) of the spectra, but not the shape. Normalizing the spectra by the variance, however, has removed this offset in Fig. 11. Fits of the Poisson model to the data show that it adequately describes the number of 'bright' pixels (defined as $>2$ fluorescence units) in any 64 pixel row or column of each subimage. It is thus reasonable to conclude that the spatial spectra are generated by isolated brightly fluorescing particles, randomly arranged in space. Certainly this is consistent with the visual impression of the images (e.g., Fig. 9).

The spatial arrangement of particles is random only on the scales of individual images $(70 \times 70 \mathrm{~cm})$, and in some cases non-random particle distributions were observed within an image (top-to-bottom gradients in the numbers of particles). On the larger vertical scale of our vertical profiles (30 to $68 \mathrm{~m}$ depth), the numbers of particles follow the intensity of fluorescence with depth - the highest numbers of particles occur at the fluorescence maxima.

The bright fluorescent particles that dominate our images are probably either aggregates of phytoplankton, or herbivores with guts full of undigested phytoplankton, or possibly both. The particles are smaller than $0.67 \mathrm{~cm}$, which is a reasonable size for an aggregate or a copepod. MacIntyre et al. (1995) report aggregates of 0.1 to $2 \mathrm{~mm}$ in diameter, although Riebesell (1992) described comet-like 'stringers' which were 10 s of centimeters long but only a few centimeters wide. These aggregates can sink rapidly, be neutrally buoyant, or even float if they contain trapped gas bubbles. MacIntyre et al. documented enhanced concentrations of aggregates at density discontinuities and inferred that changes in sinking velocity and turbulence-driven aggregation were contributing to these layers. Alldredge et al. (1990) report that turbulence dissipation rates of $10^{-7} \mathrm{~m}^{2} \mathrm{~s}^{-3}$ (the lowest values they measured) would disaggregate phytoplanktonic flocs (Chaetoceros spp. and Nitzschia spp.) of $\sim 1 \mathrm{~cm}$ diameter (the maximal sizes they examined), consistent with brightly fluorescent particles $<0.7 \mathrm{~cm}$ in diameter as inferred from our data.

To give a rough count of the number of particles in an image, a particle edge was defined as twice the modal fluorescence for each image. The number of regions with closed contours of this fluorescence value 
was enumerated in an $80 \times 80$ pixel subimage of the full image. Given that particles may be too close together to distinguish by this criterion, the particle count is probably conservative. The numbers of particles closely followed the chlorophyll fluorescence profile. Above the primary chlorophyll maximum there were 0 to 20 particles $\mathrm{l}^{-1}$. In the primary chlorophyll maximum there were about 15 to $30 \mathrm{l}^{-1}$, and 30 to $60 \mathrm{l}^{-1}$ in the secondary maximum. Fluorescent particles were rare below the secondary maximum $\left(<1 \mathrm{l}^{-1}\right)$, possibly due to reduced fluorescence per particle rather than an absence of particles. Between the primary and secondary chlorophyll maxima, the number of particles fell to $<15 \mathrm{l}^{-1}$ at about $58 \mathrm{~m}$. These numbers are consistent with literature values: for aggregates $>0.5 \mathrm{~mm}$ in diameter concentrations ranged from 10 to $600 \mathrm{l}^{-1}$ (Gorsky et al. 1992, MacIntyre et al. 1995, Kiørboe et al. 1998), while aggregates $>3 \mathrm{~mm}$ tend to be more rare: 1 to $10 \mathrm{l}^{-1}$ (e.g., Alldredge \& Silver 1988). On the other hand, these particle concentrations are much higher than would be expected for densities of copepods, supporting the contention that most of them are marine snow aggregates.

The 2 to $3 \mathrm{~m}$ thickness of the secondary chlorophyll maximum makes it slightly thicker than the usual definition of a 'thin layer' ( $<1 \mathrm{~m}$ thick; Cowles \& Donaghay 1998). This layer is not a homogeneous sheet, as layers tend to be envisioned (e.g., Franks 1995; 'Oceanography' special issue on thin layers, Vol. 11(1), 1998), but a layer with a great deal of microscale structure formed by individual particles. The layer could have formed through local growth of the particles, or accumulation of the particles through sinking and aggregation at an isopycnal (cf. Derenbach et al. 1979, MacIntyre et al. 1995). At the resolution of our data, the layer does not seem to be inclined across isopycnals, and its thin vertical extent is probably not due to vertical shearing of an initially thicker layer (cf. mechanism of Franks 1995). One scenario consistent with the data is that aggregates formed in and above the primary chlorophyll maximum ( 30 to $55 \mathrm{~m}$ ), and then sank and accumulated at the secondary maximum (cf. MacIntyre et al. 1995). The relative absence of fluorescent particles below $68 \mathrm{~m}$ could be due to a reduction in the number of particles, or a marked reduction in fluorescence of the particles forming the aggregates. This sinking scenario may also explain the lack of acoustic targets at the depth of the highest chlorophyll fluorescence (Jaffe et al. 1998): the aggregates forming the secondary maximum may be relatively unpalatable to the herbivores. Unfortunately, it is not possible to test this (or other) hypotheses with the data at hand.

The patchiness of fluorescence may also represent patchiness of physiological state of the phytoplankton, rather than variance of biomass on those scales. Given the many factors that control fluorescence yield in phytoplankton, it is entirely possible that the variability we have measured represents the scales of phytoplankton physiological or photosynthetic variability. An instrument such as the fast repetition-rate fluorometer (FRRF: Kolber et al. 1998) that could resolve these spatial scales could help to test such a hypothesis.

It is likely that some of the strongly fluorescing particles were crustacean herbivores, and it is possible that an unexpected benefit of the OSST system will be an ability to quantify herbivore gut fluorescence. To distinguish free phytoplankton fluorescence from copepod gut fluorescence will require modifications to the OSST system, including higher magnification or the sensing of additional wavelengths (for example backscattered light).

The lack of a spectral slope in our average spatial spectrum was somewhat surprising to us, given the literature suggesting that a $-5 / 3$ slope would be a likely observation (e.g., Batchelor 1959, Denman \& Platt 1976, Denman et al. 1977, Gargett 1985). These models explore continuous tracers with and without growth in a 3-dimensional turbulent field. Variance is created at large scales and dissipated at small scales around the inertial subrange of turbulent mixing. Based on physical observations, we feel that we have resolved the scales over which we would expect to see an inertial subrange, but we see no evidence of a $-5 / 3$ slope (or any slope) to the average fluorescence spectrum. Rather, the spectra are dominated by the contribution of relatively rare, isolated, randomly distributed particles - a quantum phenomenon rather than a continuum phenomenon (see also Siegel 1998). In retrospect, this should not be so surprising, as a flat spectrum would be predicted in a region dominated by aggregates. The question remains, then, whether a $-5 / 3$ slope would be found in a region without the larger particulate fluorescence, or with a higher number of particles, and what sort of sampling and statistics would be required to resolve the spectrum in a region of weak, intermittent turbulence.

\section{Potential applications of OSST}

Images of the type described here will considerably expand the field of study of microscale patchiness of plankton. Combined with appropriate physical measurements (vertical profiles of dissipation of turbulent kinetic energy and dissipation of temperature variance), the spatial information contained in these images will allow testing of hypotheses of physical and biological control of microscale structures of the phytoplankton. Theoretical microscale spatial spectra of phytoplank- 
ton have included the dynamics of a passive tracer in a turbulent field (e.g., Hinze 1959), simple growth and mixing (Denman \& Platt 1976, Denman et al. 1977) and growth with mixing and species interactions (grazing and density dependence) (Powell \& Okubo 1994). These hypothetical spectra have remained largely untested on the appropriate scales of isotropic turbulence $(<1 \mathrm{~m}$, e.g., Gargett 1985) mainly due to technological limitations. The deployment of the OSST in a variety of turbulence regimes (wind-driven, tidally driven, internalwave driven, etc.) will allow strong testing of these models, and development of new models should existing ones be rejected.

Testing of such models will require accurate data on the microscale physical structures and dynamics of the environment. Enhancing the OSST with microstructure sensors would enable coincident measurement of essential physical and biological variables at similar spatial scales. Such physical measurements will significantly enhance our ability to quantify the temporal and spatial patchiness of the microscale environment that the plankton inhabit, and how variability at these scales influences growth and trophic transfers in the plankton.

The observations of phytoplankton patchiness by OSST can also be used to explore questions of biological aggregation. This issue has received considerable attention recently (e.g., papers in 'Deep-Sea Res' II 42(1)1995), and a great deal of effort has been spent trying to understand the dynamics of aggregation, flocculation and particle stickiness in relation to phytoplankton species and densities. Sequences of OSST images taken on successive profiles through the development and decay of a bloom could help to answer questions concerning the dynamics of aggregate formation and the fate of primary production in the oceans.

While numerous laboratory experiments have been performed examining the behavioral response of herbivores to patchiness of phytoplankton (e.g., Tiselius 1992, Saiz et al. 1993), little in situ work has been done. Little is known about the microscale structure of the phytoplankton (and by inference the food environment of the herbivores), and our knowledge of how herbivores navigate within this environment is poor. The OSST will allow investigations of phytoplanktonherbivore interactions on the foraging scales of the organisms, giving a novel and important new view of trophic interactions in the sea.

Acknowledgements. This project would not have been successful without the help of many dedicated people. Particular thanks are due to Greg Adelman and Ed Reuss who did the bulk of the engineering on the OSST and FishTV systems. Mary Sisti cheerfully organized much of the cruise. Cap- tain Louis Zimm artfully piloted the RV 'Sproul', and Tammi Koonce, Bob Wilson and Fred Uhlmann were indispensable help in deploying the system. Andrew Leising, Alex DeRobertis and Adrienne Huston were a great help in sampling, and Dave Zawada processed many of the raw images. Very useful comments on the manuscript were made by James Pringle and Thomas Kiørboe. Thanks particularly to Eric Shulenberger, Ron Tipper, Steve Ackleson and Larry Clark for encouraging this enterprise.

\section{LITERATURE CITED}

Alldredge AL, Silver MW (1988) Characteristics, dynamics and significance of marine snow. Prog Oceanogr 20:41-82

Alldredge AL, Granata TC, Gotschalk CC, Dickey TD (1990) The physical strength of marine snow and its implications for particle disaggregation in the ocean. Limnol Oceanogr 35:1415-1428

Batchelor GK (1959) Small-scale variation of convected quantities like temperature in turbulent fluid. J Fluid Mech 5: 113-133

Bird JL, Kitting CL (1982) Laboratory studies of a marine copepod (Temora turbinata Dana) tracking dinoflagellate migrations in a miniature water column. Contrib Mar Sci 25:27-44

Bjornsen PK, Nielsen TG (1991) Decimeter scale heterogeneity in the plankton during a pycnocline bloom of Gyrodinium aureolum. Mar Ecol Prog Ser 73:263-267

Bohrer RN (1980) Experimental studies on diel vertical migration. In: Kerfoot W (ed) Evolution and ecology of zooplankton communities. University Press of England, London, p 111-121

Cowles TJ, Donaghay P (1998) Thin layers: observations of small-scale patterns and processes in the upper ocean Oceanography 11:2

Cowles TJ, Fessenden LM (1995) Copepod grazing and finescale distribution patterns during the marine light-mixed Layers experiment. J Geophys Res 100:6677-6686

Cowles TJ, Desiderio RA, Neuer S (1993) In situ characterization of phytoplankton from vertical profiles of fluorescence emission spectra. Mar Biol 115:217-222

Derenbach JB, Astheimer H, Hansen HP, Leach H (1979) Vertical microscale distribution of phytoplankton in relation to the thermocline. Mar Ecol Prog Ser 1:187-193

Denman KL, Platt T (1976) The variance spectrum of phytoplankton in a turbulent ocean. J Mar Res 34:593-601

Denman KL, Okubo A, Platt T (1977) The chlorophyll fluctuation spectrum in the sea. Limnol Oceanogr 22:1033-1038

Desiderio RA, Cowles TJ, Moum JN, Myrick M (1993) Microstructure profiles of laser-induced chlorophyll fluorescence spectra: evaluation of backscatter and forward-scatter fiber-optic sensors. J Atmos Oceanic Technol 10:209-224

Franks PJS (1995) Thin layers of phytoplankton: a model of formation by near-inertial wave shear. Deep-Sea Res I 42:75-91

Gargett AE (1985) Evolution of scalar spectra with the decay of turbulence in a stratified fluid. J Fluid Mech 159: 379-407

Gorsky G, Aldorf C, Kage M, Picheral M, Garcia Y, Favole J (1992) Vertical-distribution of suspended aggregates determined by a new underwater video profiler. Ann Inst Océanogr 68:275-280

Hinze JO (1959) Turbulence. McGraw Hill, New York

Jaffe JS (1999) Target localization for a three-dimensional multibeam sonar imaging system. J Acoust Soc Am 105: $3168-3175$ 
Jaffe JS, Ruess E, McGeehee D, Chandran G (1995) FTV-a sonar for tracking macrozooplankton in 3 dimensions. Deep-Sea Res I 42:1495-1512

Jaffe JS, Franks PJS, Leising AW (1998) Simultaneous imaging of phytoplankton and zooplankton distributions. Oceanography 11:24-29

Kiørboe T, Tiselius P, Mitchell-Innes B, Hansen JLS, Visser AW, Mari X (1998) Intensive aggregate formation with low vertical flux during an upwelling-induced diatom bloom. Limnol Oceanogr 43:104-116

Kolber ZS, Prasil O, Falkowski PG (1998) Measurements of variable chlorophyll fluorescence using fast repetition rate techniques: defining methodology and experimental protocols. Biochim Biophys Acta-Bioenergetics 1367:88-106

MacIntyre S, Alldredge AL, Gotschalk CC (1995) Accumulation of marine snow at density discontinuities in the water column. Limnol Oceanogr 40:449-468

McGeehee D, Jaffe JS (1996) Three-dimensional swimming behavior of individual zooplankters: observations using the acoustical imaging system FishTV. ICES J Mar Sci 53: 363-369

Morel A (1988) Optical modeling of the upper ocean in relation to its biogenous matter content (Case I waters). J Geophys Res 93:10749-10768

Owen RW (1989) Microscale and finescale variations of small plankton in coastal and pelagic environments J Mar Res 47:197-240

Palowitch AW, Jaffe JS (1994) Three-dimensional ocean chlorophyll distributions from underwater serial-sectioned fluorescence images. Appl Optics 33:3023-3033

Editorial responsibility: Otto Kinne (Editor),

Oldendorf/Luhe, Germany
Palowitch AW, Jaffe JS (1995) Optical serial sectioned chlorophyll-a microstructure. J Geophys Res 100:13267-13278

Powell TM, Okubo A (1994) Turbulence, diffusion and patchiness in the sea. Philos Trans R Soc Lond B Biol Sci 343: $11-18$

Price H (1989) Swimming behavior of krill in response to algal patches: a mesocosm study. Limnol Oceanogr 34: 649-659

Riebesell U (1992) The formation of large marine snow and its sustained residence in surface waters Limnol Oceanogr $37: 63-76$

Saiz E, Tiselius P, Jonsson PR, Verity P, Paffenhöfer GA (1993) Experimental records of the effects of food patchiness and predation on egg production of Acartia tonsa. Limnol Oceanogr 38:280-289

Siegel DA (1998) Resource competition in a discrete environment: why are plankton distributions paradoxical? Limnol Oceanogr 43:1133-1146

Sutton DW, Jaffe JS (1992) Acoustic bedload velocity estimates using a broadband pulse-pulse time correlation technique. J Acoust Soc Am 92:1692-1698

Tiselius P (1992) Behavior of Acartia tonsa in patchy food environments Limnol Oceanogr 37:1640-1651

Tiselius P, Nielsen G, Nielsen TG (1994) Microscale patchiness of plankton within a sharp pycnocline. J Plankton Res 16:543-554

Welch PD (1967) The use of fast Fourier transform for the estimation of power spectra: a method based on time averaging over short modified periodograms. IEEE (Inst Elect Electron Eng) Trans Audio Electroacoust 15:70-73

Submitted: July 13, 2000; Accepted: December 12, 2000

Proofs received from author(s): August 29, 2001 